UDC 159.922.7:376:615.851.3-053.2

\title{
FULFILLING A NEED IN ARMENIA: THE CREATION OF THE OCCUPATIONAL THERAPY GUIDEBOOK FOR INCLUSION
}

\author{
AUTHORS' DATA: \\ Victoria Babikian, MS, OTR/L \\ Premier HealthCare \\ Occupational Therapist, MS in Occupational Therapy, OTR/L \\ Contacts: BabikianOTRL@gmail.com
}

\begin{abstract}
:
In Armenia children with disabilities are often stigmatized and separated from the community. Some are sent to institutions or orphanages, some are hidden in the home, and others are hastily placed into schools and ignored in classrooms. However, Armenia is moving towards a more inclusive society. Victoria Armineh Babikian volunteered with Children of Armenia Charitable Fund (COAF) in 2017; she was their first ever Occupational Therapist. She introduced a different perspective- providing holistic care by addressing self-care needs and enhancing physical, cognitive, and sensory skills for children with intellectual and developmental disabilities. Upon completion of her volunteer experience, Babikian needed a sustainable approach to continue increasing knowledge on the care of children with disabilities. Therefore, she compiled the Occupational Therapy Guidebook to Inclusion, an 80-page manual that provides clear information about common diagnoses, simple exercises, and functional activities using homemade tools to enhance strength, coordination, cognitive processing, sensory regulation, and behavior among children with disabilities. This guidebook will be published and distributed throughout Armenia.

The purpose of the Occupational Therapy Guidebook to Inclusion is to enhance disability resources for every region of Armenia, especially within rural communities.
\end{abstract}

Keywords: Occupational therapy, international, inclusion, inclusive education, disability, volunteer, development, pediatrics. 


\section{INTRODUCTION}

In 2017, Victoria Armineh Babikian volunteered in Armenia for eight months with Children of Armenia Charitable Fund (COAF) providing occupational therapy to children with disabilities in rural villages. During her experience, she noticed there were children with disabilities kept in their homes and hidden from the community due to shame and unacceptance. There were teachers who ignored children with special needs in their class of 30+ young students. There were therapists working with complex cases who were only addressing one need or performance area. And there were parents and grandparents who knew their child needed extra help but did not know what was the first step. Babikian's aim was to answer the following questions: What services are already provided? What services are lacking? How can occupational Therapy (OT) contribute to this team and to the village?

Although there are steps towards inclusive education in the capital city such as resource rooms and visiting ABA/OT therapy, the rural regions are still transitioning. Services are scarce in these areas, and non-profit organizations such as COAF have stepped in to provide teacher training, accommodations, and family services for children with special needs. COAF advocates for these children and works with families to provide free social work services to individuals and families, and has partnered with many schools to provide clinicians for weekly speech therapy and psychology services as well as after-school tutoring for children with learning difficulties. They filled a gap in the rural Armenian education system by initiating disability services. However, it was apparent that these children would also benefit from occupational therapy, the holistic approach that addresses self-care needs and enhances physical, cognitive, and sensory skills, to address performance and participation in all occupations or activities of daily living.

The first mission was spreading awareness of occupational therapy. How would people collaborate with an OT if they did not even know what they do? Babikian presented at an annual staff development conference to introduce the field to the psychologists, social workers, afterschool teachers, and speech therapists from the 11 regions of the country. She was able to provide a deeper understanding of the training and approach to assessments, and demonstrate activities or adaptations provided. There were many professionals who identified a lack of resources for school-age children with developmental disabilities; they were receptive to incorporate recommendations and strategies into the program and wanted the new OT to visit their respective regions. Babikian found the way to fit into the team and provide a unique service. 
Armineh developed a systematic approach to evaluating children and documenting progress. This included a referral form, an occupational therapy evaluation, and daily progress note templates. The evaluation form assessed the child's current levels of cognition, visual motor and perceptual skills, fine and gross motor skills, sensory processing and occupational performance. This was used to measure baseline performance at the start of treatment, as well as to monitor progress at the final session.

The Person-Environment-Occupation Model guided Babikian's planning and program development to provide client-centered care in the rural villages. She looked at the impact of the person, environment, and occupation on performance and daily activities (Law, Cooper, Strong, Stewart et al., 1996). Below is a real example to demonstrate how to PEO Model guided Armineh's evaluation and treatment.

- Person: George is an 8-year-old boy with Down's Syndrome in the second grade. He has muscle weakness, decreased fine and gross motor coordination, visual perception, attention, and memory. George is a very happy and sociable child who enjoys going to school and playing soccer.

\section{- Environment:}

- Culture: Armenia is a collectivist community; grandparents, parents, kids, and cousins all live together and are expected to take care of each other. Individualism and independence are not valued as much. In the rural villages, this is even more apparent as resources are scarce; neighbors and families need this support in order to survive. George lives with his mother, father, paternal grandmother, and sister. Their cousins, aunts, and uncles also live in the village.

- Family: There is a strong stigma against people with disabilities. Community integration is not valued for children with special needs. George's father believed his child cannot learn and did not want to send him to school for fear of other villagers knowing about George's diagnosis. The grandmother approached COAF asking for help. COAF advocated for George and got the family to send him to school. He started when he was 7 years old, and now receives psychology, speech therapy, and after-school tutoring with COAF. COAF also provides social work and medical services for the family. 
- Language: In Armenia families speak Eastern Armenian, a dialect unique to people from Armenia. It has a different accent, conjunctions, and vocabulary from Western Armenian, which is what Armineh spoke as diasporan. This made both expressive and receptive communication a challenge. She had to learn Eastern Armenian for George to understand her, as he had never met an Armenian from another country before.

○ Schools: Schools in the villages are in poor condition. Many lack running water. There are frequent electricity cuts and school closures in the winter because of a lack of heat in the classrooms. This made it a challenge to have regular weekly sessions.

\section{- Occupations:}

- Education: George is able to recognize some numbers and can write his first name. However, he is behind in his classes, especially reading and mathematics. His second-grade peers are completing word problems while he cannot find the numbered pages in the textbook. Often times he was observed drawing in his notebook because he could not follow the teacher's directives.

- ADLs/IADLs: George's mother did everything for him. As a result, he required maximum assistance with basic self-care skills (i.e. toileting, dressing, hygiene, bathing). During school hours, the teacher called his mother to come to school to take him to the toilet. Because the toilets in the school are broken, his mother carried him over the toilet, then wiped and dressed him. He frequently has accidents and his mother has to come to school to change him.

○ Play/Leisure: The children in the village are often found playing in the dirt roads picking fruit trees, playing soccer, or jumping around the school's playground. Before attending school, George did not have many friends. He is now well-known throughout the school and enjoys playing sports with neighboring children.

For intervention, both direct and indirect service models were utilized. In eight villages closest to the capital city, individual OT sessions were provided weekly. The intervention approach required a unique balance between respecting the culture and educating about the importance of independence as the child develops. Most of the activities were inspired by items found in the home or school. For example, threading beads onto raw spaghetti for fine motor 
skills, drawing in shaving cream for tactile exploration, creating obstacle courses from school playgrounds, entrance ramps, and stairs for strengthening and coordination. Caregiver education was also provided about the services and adaptations available to children with special needs. Handouts were given with home exercises and pictured sequences for self-care skills such as dressing, toileting, tooth-brushing. Babikian also hosted a teacher in-service about inclusive education and the importance of adaptations and modifications to enhance student participation and school performance.

Consultative services were provided in further regions. This included addressing areas of concern identified by caregivers and professionals, providing functional intervention techniques, recommendations, or handmade adaptations for the local speech therapists, after-school teachers, or psychologists to incorporate OT strategies into their sessions.

After seven months of intervention, many of the children demonstrated progress in schoolrelated skills. COAF understood the scope of occupational therapy and valued its contribution to child development. Financial restrictions did not allow for the organization to hire an occupational therapist upon Armineh's departure. However, Babikian saw firsthand the outcome of her efforts. Parents, teachers, and clinicians felt more equipped to address special needs, children were receiving individualized care. This previously ignored population was now more independent in daily activities, were included in community events, and had improved quality of life.

Upon completion of her volunteer experience, Babikian wanted a sustainable approach to be able to continue this work; she knew that annual visits or intermittent workshops would not be sufficient. Thus, she decided to create the Occupational Therapy Guidebook to Inclusion. This guidebook includes clear information about common diagnoses, simple exercises, and functional activities using homemade tools to enhance strength, coordination, cognitive processing, sensory regulation, and behavior among children with disabilities. The purpose of the guidebook is to increase the understanding of this population and to help caregivers provide early intervention and holistic care across all settings. The Occupational Therapy Guidebook to Inclusion can help increase resources for disability services, promote inclusion for children with disabilities and enhance their independence in the home, school, and community. 


\section{THE BODY}

Children with disabilities are often viewed as bystanders, objects of pity or charity, and ostracized from communities. This mentality was strongly promoted within the Soviet Union as there was a large emphasis on uniformity. Many educators would claim, "There are no invalids in the USSR!" They were opposed to inclusion, stating that "the mentally retarded . . . cannot be builders or creators of a new life," and the most that could be demanded from such children is that they "not keep others from building" (Vygotsky, 2012).

Since the fall of the Soviet Union, Armenia has been attempting to shift their mentality towards inclusion. In 2006, the United Nations compiled a protocol, Convention on the Rights of Persons with Disabilities, to change global attitudes and approaches towards this population. It emphasized that individuals with disabilities have rights, are capable of making decisions, and should be active members of society. Armenia signed the Convention in 2007 with formal ratification in 2010 (UN CRPD, 2019). However, implementation has been a struggle. There are approximately 200,000 individuals with disabilities in Armenia. Thousands of disables children are institutionalized even though more than 90\% have at least one living parent (HRW, 2017; HRW, 2018; HRW, 2018). Less than $20 \%$ have completed secondary education, compared to $84 \%$ of the overall population. Over $90 \%$ of adults with disabilities are unemployed, compared to $18 \%$ of the general population (Mijatović, 2018). It is evident that this population is greatly underserved because of stigmatization and limited resources for educators, clinicians, and service providers to properly address the needs of Armenians with disabilities.

Armineh Babikian witnessed this issue when she was a Birthright Armenia volunteer in 2017 with Children of Armenia Charitable Fund (COAF). She met many families who were ashamed of their non-typical offspring and required strong advocacy to send them to school. Then the children were placed into classrooms with teachers who were not trained to address special needs. This resulted in a gap in services, as well as a lack of appropriate support, education, and opportunity for these children. Babikian believed that occupational therapy can help bridge the gap, as this unique profession is involved in education, rehabilitation, and home-care settings.

Occupational therapy (OT) helps individuals become independent in any activity that "occupies" their time. This can range from basic self-care tasks such as getting dressed, to playing sports, writing homework, or performing one's job. An OT (1) assesses the individual's lifestyle, roles, and routines, (2) addresses the physical, psychosocial, and sensory skills required to perform daily 
tasks, and (3) analyzes the environmental accessibility and safety. The clinician then provides individualized therapy or adaptations for optimal independence. Occupational therapists work in hospitals, long-term care facilities, rehabilitation or developmental centers, schools, and in the home. They provide a valuable service that promotes inclusion and quality of life for individuals with disabilities. However, this is a small field in Armenia. Khachatur Abovyan Armenian State Pedagogical University houses the only OT program in the nation. There are approximately 100 occupational therapists, or "ergotherapists," throughout Armenia. Most are employed in Yerevan and there is a small portion in Gyumri; thus rural regions are left without this crucial service. As a result, there are many children relying on caregivers for maximum assistance, parents who are often exhausted and frustrated, and teachers who feel overwhelmed because they do not have the right tools to serve this population.

The Occupational Therapy Guidebook to Inclusion can add to this toolkit and increase disability resources. The guidebook provides simple clear information about common diagnoses, exercises with diagrams to strengthen physical, cognitive, sensory processing, and behavioral skills, as well as practical classroom strategies. It aims to enhance participation in school, selfcare, leisure activities, and community integration for the children of Armenia. All tools and activities described can be made with simple household items. The Guidebook also includes worksheets which can be photocopied and a resource library of Armenian disability services. This guidebook will be distributed for free to all who care for or work with children with disabilities. In addition, free workshops will be hosted in each region at local developmental centers and schools to ensure comprehension, demonstrate exercises, and answer specific questions.

The emphasis of this Guidebook in inclusion. By spreading knowledge and strategies, the author aims to decrease occupational injustice for children with disabilities in Armenia. Occupational injustice can come in four forms: occupational alienation, deprivation, imbalance, and marginalization. Inclusion can decrease alienation, allow for participation and balance in activities, and remove marginalization by providing opportunity for this population (Townsend, 2012). In fact, everyone benefits from inclusion. Research shows that inclusion can lead to better learning outcomes for all children, not just children with disabilities. It promotes tolerance, enables social cohesion, and provides equal participation in society. Financially, inclusion is more cost effective than separated schooling and produces inclusive labor markets which lead to a more efficient social economy (UNICEF, 2017). Ultimately everyone benefits from inclusion! 
Therefore, this guidebook can not only help caregivers of children with disabilities, but also the greater public of Armenia.

\section{ANSWER/CONCLUSION/SUMMARY:}

The Occupational Therapy Guidebook to Inclusion will provide holistic occupational therapy strategies that caregivers, teachers, and clinicians can utilize with children with various developmental, intellectual, or physical disabilities in order to increase independence and quality of life. It can promote inclusion, decrease stigma, and provide new opportunities for Armenian children with disabilities.

\section{REFERENCE LIST}

1. Human Rights Watch. (2018). "Armenia: Children isolated, needlessly separated families." Retrieved from

2. https://www.hrw.org/news/2017/02/22/armenia-children-isolated-needlessly-separatedfamilies

3. Human Rights Watch. (2018). "When Will I Get to Go Home?" | Abuses and Discrimination against Children in Institutions and Lack of Access to Quality Inclusive Education in Armenia. Retrieved from https://www.hrw.org/report/2017/02/22/when-willi-get-go-home/abuses-and-discrimination-against-children-institutions

4. Law, M., Cooper, B. A., Strong, S., Stewart, D., Rigby, P., \& Letts, L. (1996). The personenvironment-occupation model: A transactive approach to occupational performance. Canadian Journal of Occupational Therapy, 63, 9-23.

5. Mijatović, D. (2018). Upholding the human rights of persons with disabilities in Armenia. Retrieved from https://www.coe.int/en/web/commissioner/-/upholding-the-human-rightsof-persons-with-disabilities-in-armenia

6. Townsend, E. A. (2012). Boundaries and bridges to adult mental health: Critical occupational and capabilities perspectives of justice. Journal of Occupational Science, 19, 8-24.

7. UNICEF. (2017). Inclusive education- Including children with disabilities in quality learning: What needs to be done? Retrieved from 
https://www.unicef.org/eca/sites/unicef.org.eca/files/IE_summary_accessible_220917_bri ef.pdf

8. United Nations. (2019). 15. Convention on the Rights of Persons with Disabilities. Retrieved from https://treaties.un.org/Pages/ViewDetails.aspx?src=TREATY\&mtdsg_no=IV$15 \&$ chapter $=4 \&$ clang $=\_$en

9. Vygostsky, L. S. (2012). The Collected Works of L.s. Vygotsky The Fundamentals of Defectology (Abnormal Psychology and Learning Disabilities). Retrieved from https://books.google.com/books?id=jUgyBwAAQBAJ\&dq=.\&source=gbs_navlinks 